\title{
D-Dimer Levels In Breast Carcinoma: A Clinico-Pathologic Study
}

\author{
Sapna Patel ${ }^{1}$, Rashmi $C^{1 *}$ and Harish $\mathrm{S}^{2}$
}

${ }^{1}$ Department of Pathology, JSS Medical College, JSS University, Sri Shivarathreeshwara Nagar, Mysuru-570015, Karnataka, India ${ }^{2}$ Department of surgery, JSS Medical College, JSS University, Sri Shivarathreeshwara Nagar, Mysuru-570015, Karnataka, India

\begin{abstract}
Background: Breast cancer is the most common cause of death in women by neoplasia. Plasma D-dimer is a hypercoagulability and fibrinolytic system marker, which is produced when factor XIIIa (a cross-linked fibrin) is degraded by plasmin. It is increased in various solid tumour patients including breast, lung, prostate, cervical and colorectal cancers.

Methods: 30 patients of breast carcinoma who underwent radical mastectomy (cases) and 30 cases without carcinoma (controls) were included. The baseline parameters (D-dimer levels) were measured in both cases and controls. From each patient, whole blood was collected and Quantitative D-dimer levels were obtained by Diazyme's D-dimer Assay using the instrument, RANDOX Rx imola. Other parameters such as histopathological features, hormonal receptors (ER \& PR) and HER2/neu status were studied in cases.

Result: Mean plasma D-dimer levels were significantly higher in patients with breast carcinoma before surgery as compared to after surgery $(p<0.001)$. Quantitative D-dimer levels highly correlated with lymph node status and histopathological grading $(p=0.003$, $p=0.015$ respectively).

Conclusion: Our study showed that high plasma D-dimer levels can be used as a marker for lymph node involvement and higher histopathological grade. Due to the ease with which plasma D-dimer levels can be obtained and its cost effectiveness, quantitative D-dimer levels can be added to models for predicting axillary lymph node involvement.
\end{abstract}

Keywords: Breast Carcinoma, Fibrinolytic System, Axillary Lymph Node, D-Dimer, Histopathological Grading.

\section{Introduction}

Breast cancer is the most common cancer in women and accounts for $29 \%$ of all cancers diagnosed each year worldwide. In India, it is the second most common cancer after cervical cancer. The involvement of axillary lymph nodes, larger size of the tumour, hormone receptor negativity, presence of the mutation of p53 and vascular invasion determine poor prognosis and treatment options upon initial diagnosis. ${ }^{[1,2]}$ There is evidence that the components of the coagulation / fibrinolytic system play a role in cancer biology and angiogenesis. At the time of diagnosis, majority of the cancer patients have laboratory evidence of systemic coagulation activation. The activated coagulation system makes the disease more aggressive and hence highlights the prognostic implications of the mediators of this mechanism. D-dimer is produced when factor XIIIa is degraded by plasmin. D-dimer levels are elevated in the plasma of various solid tumour patients including breast, lung, prostate, cervical and colorectal cancers. ${ }^{[1,3,4]}$ Currently, studies concerning the blood factors involved in angiogenesis and coagulation are scarce. Elevated levels of circulating D-dimer have been correlated with an enhanced progression of the disease and a reduced overall survival in metastatic breast cancer. In patients with operable breast cancer, D-dimer levels have been shown to be elevated at the time of diagnosis and to decrease during adjuvant chemotherapy. ${ }^{[2,4]}$ Determining lymph node status via standard lymph node dissection creates many significant long-term side effects and many attempts have been made to predict lymph node status without undergoing a full lymph node dissection. A significant relationship has been noted between presence of elevated D-dimer and involved axillary lymph nodes ${ }^{[4]}$ Overexpression of HER2/neu (human epidermal growth factor receptor 2), is associated with a poorer clinical outcome and patients with the hormone receptor negative and HER2/neu-negative status tend to present a less favourable prognosis than do those with HER2/neu-positive status. ${ }^{[1]}$

D-dimer levels have been found to be significantly increased in patients with breast cancer (cases) when compared with patients without carcinoma (controls). ${ }^{[4,5]}$ D-dimer levels correlate with clinical stage, lymphovascular invasion and axillary lymph node involvement in operable breast cancer and D-dimer was suggested to be a biomarker for predicting early tumour metastases. ${ }^{[2,6]}$ Therefore haemostatic abnormalities associated with cancer pose a 
major challenge to the clinician which may be in the form of thrombosis or excessive bleeding. ${ }^{[1,7,8]}$

\section{Materials and Methods}

The present study was undertaken in the Department of Pathology, JSS Medical College and Hospital, Mysore. Study type is prospective and included 30 cases and 30 controls between 2014-2016. Ethical clearance was obtained by the University Ethical Committee

Aims and Objectives 1; To compare the plasma D-dimer levels before and after radical mastectomy in breast carcinoma patients. 2) To assess the relationship of plasma D-dimer levels with tumour size, grade and lymph node status.

\section{Exclusion Criteria:}

Patients who had undergone lumpectomies/ invasive surgeries/ neoadjuvant chemotherapy within 3 months of the surgical (radical mastectomy) procedure.

Conditions altering the coagulation factors such as warfarin therapy, coagulation disorders, liver disease, pregnancy, major trauma and recent surgery.

Statistical Methods: The data was entered through Microsoft Excel 2007 and statistical analysis was performed using Statistical Package for Social Sciences (SPSS) version 22. Comparison of data between two independent groups was made by independent samples $t$ test, between three or more independent groups by ANOVA (Analysis of variance), between pre-operative and post-operative D-dimer values as well as between cases and controls by paired t-test. $p$ values $<0.05$ were considered statistically significant.

Method of Collection of Data: The baseline parameters (D-dimer levels) were measured in 30 cases and 30 controls. Other parameters such as histopathological features, estrogen receptor (ER), progesterone receptor (PR) and HER2/neu status were studied only in cases. $5 \mathrm{ml}$ of whole blood was drawn and centrifuged within 4 hours of venipuncture. Centrifuged plasma was stored at $-80^{\circ} \mathrm{C}$ until assays were run. Quantitative D-dimer levels were obtained by Diazyme's D-dimer Assay which is based on a latex enhanced immunoturbidimetric method using the instrument, RANDOX Rx imola. D-dimer levels $<0.50$ microgram/ml FEU (Fibrinogen Equivalent Units) were considered to be normal. D-dimer levels were measured once in controls and twice in cases, first prior to surgery and second 2 weeks after surgery. Surgically removed breast cancer tissues were studied. After formalin fixation, paraffin embedding and staining with hematoxylin and eosin (H\&E), histopathological features were studied.
Histopathological grade was assessed using Bloom and Richardson method, modified by Elston and Ellis. Tumour, Node, Metastasis staging (TNM staging) was used for pathological staging. Immunohistochemistry was performed on $4 \mu \mathrm{m}$ thick sections on poly-l-lysine coated slides. Antigen retrieval was done in citrate buffer at $\mathrm{pH}$ 9.2. Correlation with hormonal and HER2/neu receptors was done wherever possible.

\section{Result}

Twenty nine of the patients were female and 1 patient was male. Mean age of the patients was 46 years and median age was 50 years. Of the 30 radical mastectomy specimens, 26 were reported as Invasive Breast Carcinoma of No Special Type (NST) (Fig 1), 2 were reported as Invasive Lobular Carcinoma, 1 as Invasive Papillary Carcinoma and 1 as Medullary Carcinoma.Mean D-dimer levels during the pre-operative period ranged from $0.36 \mathrm{microgram} / \mathrm{ml} \mathrm{FEU}$ to $6.31 \mathrm{microgram} / \mathrm{ml} \mathrm{FEU} \mathrm{with} \mathrm{a} \mathrm{mean} \mathrm{of} 1.631+/-0.455$ microgram $/ \mathrm{ml} \mathrm{FEU} \mathrm{and} \mathrm{a} \mathrm{median} \mathrm{of} 1.22$ microgram $/ \mathrm{ml}$ FEU. Mean D-dimer levels during the post-operative period ranged from $0.20 \mathrm{microgram} / \mathrm{ml} \mathrm{FEU} \mathrm{to} 2.10 \mathrm{microgram} /$ $\mathrm{ml} \mathrm{FEU} \mathrm{with} \mathrm{a} \mathrm{mean} \mathrm{of} 0.669+/-0.176 \mathrm{microgram} / \mathrm{ml} \mathrm{FEU}$ and a median of 0.61 microgram $/ \mathrm{ml} \mathrm{FEU.} \mathrm{Mean} \mathrm{plasma}$ D-dimer levels were significantly higher in patients before surgery as compared to levels after surgery. This may be explained by the higher D-dimer levels in the presence of a high tumour load, which then decreases when the tumour burden is reduced by radical mastectomy.Quantitative D-dimer levels were measured in patients without carcinoma admitted in our hospital (controls). The patients were admitted for various conditions such as 6 patients with Chronic Obstructive Lung Disease (COPD) , 4 with bronchopneumonia, 2 with Systemic Lupus Erythematosus (SLE), 2 with viral fever, 2 with cellulitis and 1 each with acute appendicitis, ileal tuberculosis, tuberculosis of lung, bronchitis, febrile illness, interstitial lung disease, intestinal obstruction, nasal polyp, necrotising fasciitis, non cardiac chest pain, pleuritis, urinary tract infection (UTI), varicose veins and nephropathy. Mean D-dimer levels in the control group was found to be $0.142+/-0.071 \mathrm{microgram} / \mathrm{ml}$ FEU and the median was 0.01 microgram $/ \mathrm{ml} \mathrm{FEU.} \mathrm{Mean}$ plasma D-dimer levels were significantly higher in cases as compared to controls.Quantitative D-dimer levels highly correlated with lymph node status $(\mathrm{pN})$ and histological grading. D-dimer levels did not have any significant association with tumour size (pT), lymphovascular invasion, hormonal (ER, PR) or HER2/neu status.Majority of the cases were of pT2 i.e, 24 cases. There was 1 of pT1, 4 of pT3 and 1 of pT4 tumours. Mean D-dimer levels were found to be highest in patients with pT2 stage with 
the lowest mean D-dimer levels in pT4 stage. There was no significant correlation between D-dimer levels and tumour size (Table 1). 11 cases were staged as pN0, 11 cases as pN1, 3 cases as pN2 and 5 cases as pN3. Mean D-dimer levels were found to be highest in patients with $\mathrm{pN} 3$ and lowest in those with pN0. There was a significant correlation between lymph node stage and D-dimer levels (Table 1). We found a significant correlation between lymph node positivity and D-dimer levels (Table 1).

26 radical mastectomy specimens that were diagnosed as Invasive Breast Carcinoma of No Special Type (NST) were graded. There was a predominance of high grade tumours i.e, tumours of grade 2 and grade 3 while there were no grade 1 (well differentiated) tumours. Mean D-dimer levels were found to be higher in patients with grade 3 tumours as compared to patients with grade 2 tumours. This difference was found to be statistically significant (Table 1).

The mean D-dimer levels were found to be higher in patients with LVI when compared to those without LVI. However, this difference was not found to be statistically significant (Table 1)

ER, PR and HER2/neu status were analysed only in 14 cases. $57 \%$ of the cases ( 8 cases) were positive for estrogen receptors (Fig 2 ) and $50 \%$ of the cases ( 7 cases) were positive for progesterone receptors (Fig 3). There was no significant correlation between D-dimer levels and ER or PR status. $21.4 \%$ of cases (three cases) were positive for HER2/neu (Fig 4). Six cases were negative and five cases were inconclusive. There was no statistically significant difference among the groups. (Table 1)

Table 1: CorrelatIon of D-Dimer Levels with other Known Prognostic Factors of Breast Cancer.

\begin{tabular}{|c|c|c|c|c|}
\hline Histopathological parameters & & $\begin{array}{c}\text { Number of patients } \\
n(\%)\end{array}$ & $\begin{array}{l}\text { Mean Pre-operative D-dimer } \\
\text { level (microgram/ml FEU- } \\
\text { Fibrinogen Equivalent Units) }\end{array}$ & p Value \\
\hline Tumour size: $\mathrm{pT}$ & $\begin{array}{l}\text { T1 } \\
\text { T2 } \\
\text { T3 } \\
\text { T4 }\end{array}$ & $\begin{array}{c}1(3.3) \\
24(80.1) \\
4(13.3) \\
1(3.3)\end{array}$ & $\begin{array}{c}1.25 \\
1.67+/-1.32 \\
1.38+/-0.74 \\
0.99\end{array}$ & 0.878 \\
\hline Lymph node status: pN & $\begin{array}{l}\text { N0 } \\
\text { N1 } \\
\text { N2 } \\
\text { N3 }\end{array}$ & $\begin{array}{c}11(36.7) \\
11(36.7) \\
3(10) \\
5(16.6) \\
\end{array}$ & $\begin{array}{l}0.89+/-0.46 \\
1.68+/-0.99 \\
1.63+/-0.54 \\
3.14+/-1.78 \\
\end{array}$ & 0.003 \\
\hline Lymph node positivity & $\begin{array}{l}\text { Positive } \\
\text { Negative }\end{array}$ & $\begin{array}{l}19(63) \\
11(37)\end{array}$ & $\begin{array}{l}2.05+/-1.31 \\
0.89+/-0.46\end{array}$ & 0.009 \\
\hline Grade & $\begin{array}{l}2 \\
3 \\
\end{array}$ & $\begin{array}{l}12 \\
14 \\
\end{array}$ & $\begin{array}{l}1.07+/-0.59 \\
2.25+/-1.46 \\
\end{array}$ & 0.015 \\
\hline Lymphovascular invasion & $\begin{array}{l}\text { Absent } \\
\text { Present }\end{array}$ & $\begin{array}{l}15(50) \\
15(50)\end{array}$ & $\begin{array}{l}1.55+/-1.00 \\
1.72+/-1.43 \\
\end{array}$ & 0.705 \\
\hline ER status & $\begin{array}{c}\text { Negative } \\
\text { Positive }\end{array}$ & $\begin{array}{l}6 \\
8 \\
\end{array}$ & $\begin{array}{l}1.35+/-0.92 \\
1.05+/-0.60 \\
\end{array}$ & 0.465 \\
\hline PR status & $\begin{array}{l}\text { Negative } \\
\text { Positive }\end{array}$ & $\begin{array}{l}8 \\
6\end{array}$ & $\begin{array}{l}1.19+/-0.86 \\
1.17+/-0.62 \\
\end{array}$ & 0.980 \\
\hline HER2/neu status & $\begin{array}{l}0 \\
1 \\
2 \\
3\end{array}$ & $\begin{array}{l}3 \\
3 \\
5 \\
3\end{array}$ & $\begin{array}{l}1.83+/-1.12 \\
0.67+/-0.47 \\
0.95+/-0.29 \\
1.43+/-0.82\end{array}$ & 0.218 \\
\hline
\end{tabular}

Table 2: Comparison of D-Dimer Levels in Breast Cancer Patients with other Studies

\begin{tabular}{|l|c|c|c|}
\hline Study & $\begin{array}{c}\text { Mean D-dimer Levels } \\
\text { (microgram/ml FEU) }\end{array}$ & $\begin{array}{c}\text { Standard deviation } \\
\text { (microgram/ml FEU) }\end{array}$ \\
\hline Suega et al ${ }^{[18]}$ & 0.96 & - & - \\
\hline Dirix et al ${ }^{[9]}$ & 2.1 & 3.6 & 0.87 \\
\hline Ay et al ${ }^{[6]}$ & - & - & 0.46 \\
\hline Benoy et al ${ }^{[19]}$ & 0.57 & 0.5 & 0.42 \\
\hline Present study & 1.63 & 1.21 & 1.22 \\
\hline
\end{tabular}


Table 3: Correlation of D-Dimer Levels with Lymph Node Status

\begin{tabular}{|l|c|c|}
\hline & $\begin{array}{c}\text { Percentage of patients with positive } \\
\text { nodes (\%) }\end{array}$ & $\begin{array}{c}\text { Association with D-dimer levels } \\
(\mathbf{p} \text { value <0.05) }\end{array}$ \\
\hline${\text { Blackwell et al }{ }^{[4]}}^{[1]}$ & 35 & Yes \\
\hline Batschauer et al ${ }^{[1]}$ & 47 & No \\
\hline Oya et al $^{[8]}$ & 26 & Yes \\
\hline Kwon et al ${ }^{[21]}$ & 34.5 & Yes \\
\hline Liu et al ${ }^{[10]}$ & 68 & Yes \\
\hline Xu G et al ${ }^{[20]}$ & 57 & Yes \\
\hline Present study & 63 & \\
\hline
\end{tabular}

Table 4: Correlation of Various Histopathological Prognostic Factors with D-Dimer Levels and Comparison with other Studies

\begin{tabular}{|c|c|c|c|}
\hline Histopathological parameters & p value: Present study & p value: Blackwell et al ${ }^{[4]}$ & p value: Batschauer et al ${ }^{[1]}$ \\
\hline Tumour size & 0.878 & 0.008 & - \\
\hline Grade & 0.015 & 0.70 & - \\
\hline Lymph node positivity & 0.009 & 0.0001 & 0.260 \\
\hline Lymphovascular Invasion & 0.705 & 0.0002 & - \\
\hline ER status & 0.465 & 0.019 & - \\
\hline PR status & 0.980 & 0.73 & - \\
\hline HER2/neu status & 0.218 & - & 0.823 \\
\hline
\end{tabular}

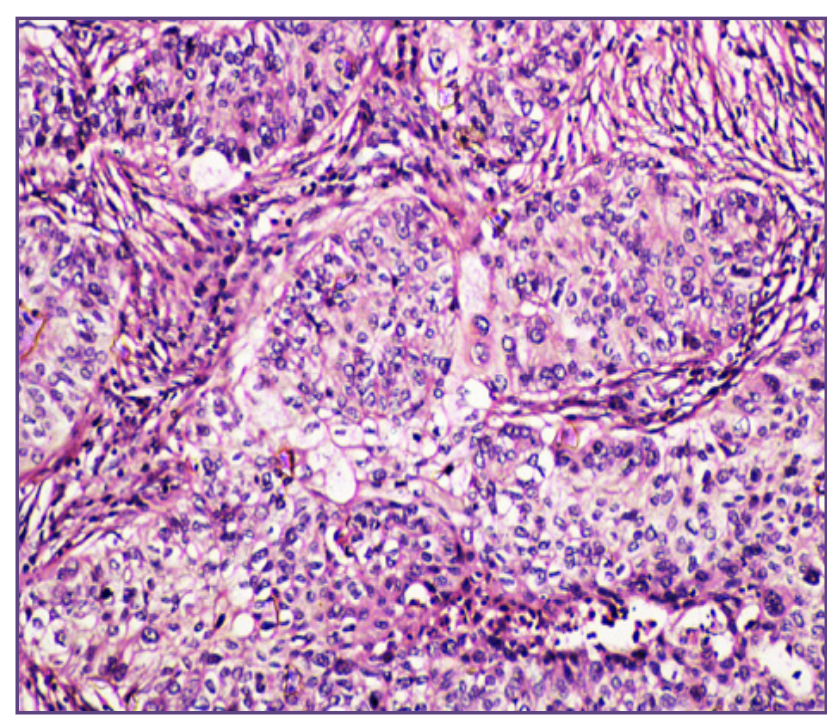

Fig. 1: Invasive Breast Carcinoma of No Special Type (NST) : Section shows pleomorphic tumour cells arranged in nests. (H\&E, x200).

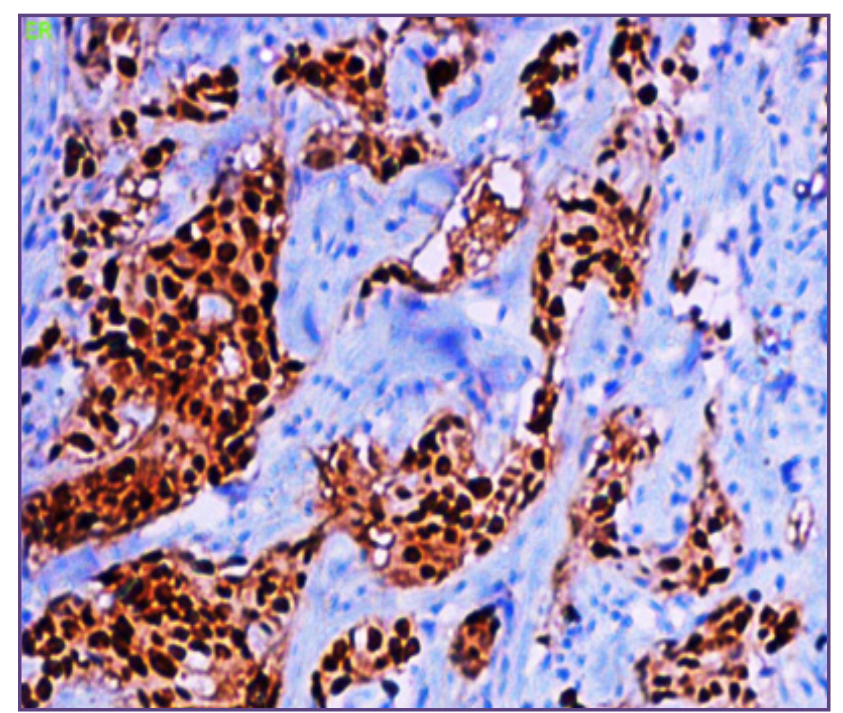

Fig. 2: ER Positive: Tumour cells showing nuclear positivity with estrogen receptor immunostain (ER IHC, DAB, x200). 


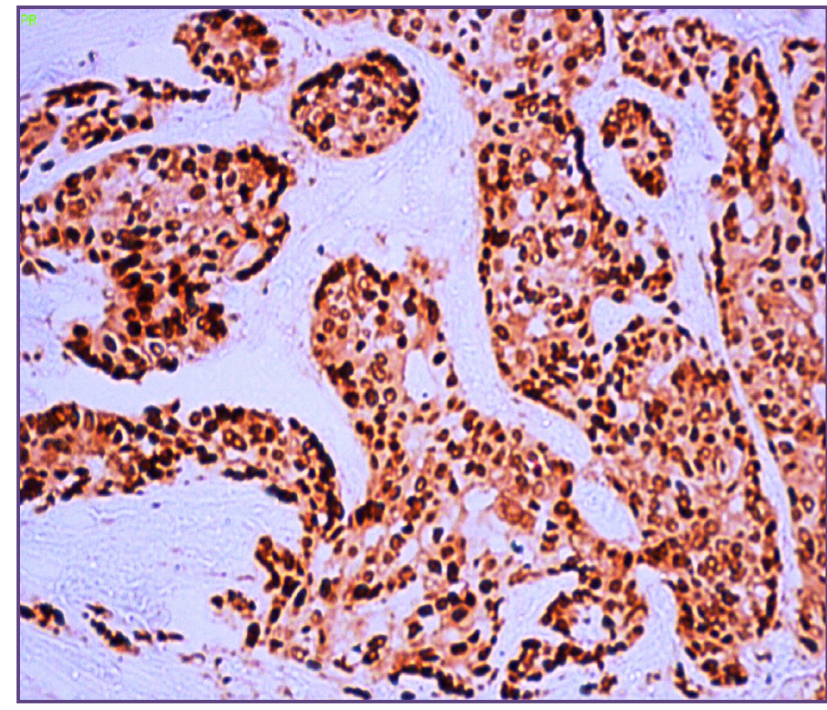

Fig. 3: PR positive : Tumour cells showing nuclear positivity with progesterone receptor immunostain (PR IHC, DAB, x200).

\section{Discussion}

The association between cancer and the activation of blood coagulation was first described more than 100 years ago. Among the degradation products resulting from the proteolytic actions of plasmin on fibrin, D-dimer is the smallest by-product, exhibiting unique characteristics. ${ }^{[9,10]}$ It is a biomarker that globally indicates the activation of hemostasis and fibrinolysis. ${ }^{[6]}$ It is a stable and sensitive degradation product of cross-linked fibrin. ${ }^{[11,12,13]}$ Metastasis is a multi-step process involved in the alterations of cellcell adhesion, angiogenesis, degradation of extracellular matrix, escape of immune surveillance and cell-matrix adhesion. ${ }^{[14]}$ Degradation of extracellular matrix is important for tumour growth and invasion, which in part is regulated by the plasminogen activation system. ${ }^{[15,16,17]}$ Therefore, an elevated plasma D-dimer level indicates activation of both coagulation and fibrinolysis. ${ }^{[8]}$

The present study showed a significant difference between D-dimer levels in breast carcinoma patients pre-operatively and post-operatively as well as significantly higher levels in cases as compared to controls. Also, D-dimer levels highly correlated with lymph node status and histopathological grading. These findings suggest that tumour progression is associated with activation of coagulation and fibrin formation, which is implicated in cancer cell proliferation as well as metastasis. ${ }^{[15]}$

D-dimer levels found in our study were found to be close to the values found in the study by Dirix et al ${ }^{[9]}$ and D-dimer levels in our study were higher than the levels found in studies done by Suega et al, ${ }^{[18]}$ Ay et al ${ }^{[3]}$ and Benoy et

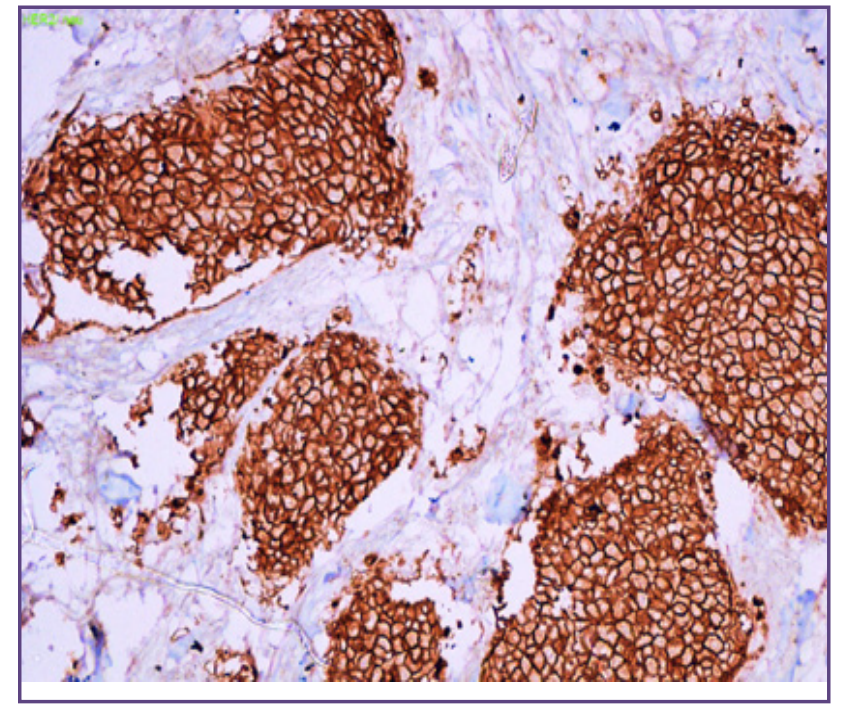

Fig. 4: HER2/neu Score 3 : Tumour cells showing strong membrane positivity with HER2/neu receptor immunostain (HER2/neu IHC, DAB, x200).

al. ${ }^{[19]}$ (Table 2)

In our study, mean plasma D-dimer levels were significantly higher in patients before surgery as compared to levels after surgery. This observation is similar to the findings of $\mathrm{Xu} \mathrm{G}$ et al ${ }^{[20]}$ who did a study on operable colorectal carcinoma patients, wherein it was observed that D-dimer levels were remarkably elevated pre-operatively and after surgery the D-dimer levels began to descend stepwise and after 2 weeks almost returned to control level. ${ }^{[20]}$ This could be due to decrease in tumour burden following surgery.

In our study, mean plasma D-dimer levels were significantly higher in cases as compared to levels in controls. This was similar to the findings by Batschauer et al, ${ }^{[1]}$ Dirix et al ${ }^{[9]}$ and Ahmed et al. ${ }^{[5]}$

In our study, we found a positive correlation between lymph node involvement and D-dimer levels. This was similar to the findings of Blackwell et al, ${ }^{[4]}$ Kwon et al ${ }^{[21]}$, Liu et al ${ }^{[10]}$ and $\mathrm{Xu} \mathrm{G}$ et al ${ }^{[20]}$. However, Batschauer et al ${ }^{[1]}$ and Oya et al ${ }^{[8]}$ found no significant association between lymph node positivity and D-dimer levels. This increase in D-dimer levels in patients with lymph node involvement could be due to fibrin formation and remodelling of the extracellular matrix which is a necessary initial step required for the cancer cells to be able to metastasize. (Table 3)

There was no significant correlation between tumour size and D-dimer levels. In the study done by Blackwell et al, ${ }^{[4]}$ they found a significant correlation between tumour size and D-dimer levels. (Table 4). The lack of correlation in our study may be explained by the fact that majority of the 
patients in our study, were of pT2 stage. This clustering of cases of similar size may be responsible for the insignificant $\mathrm{p}$ value.

There was a positive correlation between D-dimer levels and histopathological grade in the present study. However, studies done by Dirix et al ${ }^{[9]}$ and Blackwell et al ${ }^{[4]}$ found no significant association between the two. (Table 4)

Various studies have shown that D-dimer levels are increased in cancer patients as compared to the control population ${ }^{[1,5,7]}$ which was comparable to our findings of higher D-dimer levels in cases as opposed to controls. Higher levels of D-dimer have also been observed in patients with regional and distant metastatic disease, shortened survival time and higher stage. ${ }^{[10]}$ Some of the studies done on carcinoma breast that have tested for the correlation between D-dimer levels and various histopathological prognostic factors have documented varied results. ${ }^{[1,4,7]} \mathrm{In}$ our study, we found a strong correlation between D-dimer levels and lymph node involvement as well as tumour grade. Being a non-invasive and inexpensive test, D-dimer levels can be estimated with ease and is potentially a good prognostic test to be used in routine clinical practice in conjunction with other prognostic markers. However, a larger sample size and an extended follow up of patients are required to validate its use.

\section{Conclusion}

Breast carcinoma is a major cause of mortality and morbidity worldwide. There has been a shift in the trend towards breast conservative surgery as well as increasing acceptance in the omission of axillary dissection. This is because of the significant long-term side effects of complete axillary dissection such as pain, numbness and lymphedema. D-dimer levels are found to correlate significantly with lymph node status. These findings support the use of D-dimer levels as a predictive marker for lymph node metastasis. It could be used as an adjunct to other studies such as ultrasonography of axilla with or without sentinel lymph node biopsy for predicting lymph node involvement in carcinoma breast patients.

D-dimer level was found to be a prognostic parameter with high concentrations being associated with worse survival. Increased plasma D-dimers was a predictor for more rapid tumour growth, the presence of more widespread disease and for shorter survival in breast cancer patients. Considering higher levels of D-dimer in patients with a poor outcome, its evaluation may be a promising tool for prognosis in women with operable breast cancer.

\section{Acknowledgements}

Dr Akila Prashant, Associate Professor, Department of Biochemistry Vasanth, Technician, Department of Pathology, Purvachar, Technician, Department of Biochemistry

\section{Reference}

1. Batschauer AP, Figueiredo CP, Bueno EC, Ribiero MA, Dusse LMS, Fernandes AP et al. D-dimer as a possible prognostic marker of operable hormone receptor-negative breast cancer. Ann Oncol. 2010;21(6):1267-1272.

2. Khangarot SS, Gupta N, Goswami B, Hadke NS, Lal P, Gupta $\mathrm{N}$ et al. Correlation of D dimer and factor VIII levels with histopathology in patients with breast carcinoma. Cancer Biomark. 2010;7:305-314.

3. Ay C, Vormittag R, Dunkler D, Simanek R, Chiriac AL, Drach $\mathrm{J}$ et al. D-Dimer and Prothrombin Fragment $1+2$ Predict Venous Thromboembolism in Patients With Cancer: Results From the Vienna Cancer and Thrombosis Study. J Clin Oncol. 2009;27(25):4124-4129.

4. Blackwell K, Haroon Z, Broadwater G, Berry D, Harris L, Iglehart JD et al. Plasma D-dimer levels in operable breast cancer patients correlate with clinical stage and axillary lymph node status. J Clin Oncol. 2000;18(3):600-608.

5. Ahmed T, Ahmed RS, Basharat MU, Mushtaq MH, Gill SS, Khawaja AR et al. Comparative study to assess coagulation abnormalities in breast cancer. Adv.life sci. 2013;1(2):96-103.

6. Ay C, Dunkler D, Pirker R, Thaler J, Quehenberger P, Wagner $\mathrm{O}$ et al. High D-dimer levels are associated with poor prognosis in cancer patients. Haematologica. 2012;97(8):1158-1164.

7. Sitalakshmi S, Rameshkumar K, Damodar P. Significance of Haemostatic Markers in Ovarian Carcinoma. Indian J Med Paediatr Oncol. 2008;29(2):6-10.

8. Oya M, Akiyama Y, Okuyama T, Ishikawa H. High preoperative D dimer Level is Associated with Advanced Tumor Stage and Short Survival After Curative Resection in Patients with Colorectal Cancer. Jpn J Clin Oncol. 2001;31(8):388-394.

9. Dirix LY, Salgado R, Weytjens R, Colpaert C, Benoy I, Huget $\mathrm{P}$ et al. Plasma fibrin D-dimer levels correlate with tumour volume, progression rate and survival in patients with metastatic breast cancer. $\mathrm{Br} \mathrm{J}$ Cancer. 2002;86(3):389-395

10. Liu L, Zhang X, Yan B, Gu Q, Zhang X, Jiao J et al. Elevated Plasma D-Dimer Levels Correlate with Long Term Survival of Gastric Cancer Patients. PLOS ONE. 2014;9(3):e90547.

11. Alcalay A, Wun T, Khatri V, Chew HK, Harvey D, Zhou H et al. Venous thromboembolism in patients with colorectal cancer: incidence and effect on survival. J Clin Oncol. 2006, 24(7):1112-1118. 
12. Francis CW, Marder VJ. Mechanism of fibrinolysis. In: Coller B, editor. Williams hematology. 5th ed. New York: McGraw-Hill; 1995: 1252-1260.

13. Buccheri G, Torchio P, Ferrigno D. Plasma Levels of D-Dimer in Lung Carcinoma: Clinical and Prognostic Significance. Cancer. 2003;97(12):3044-3052.

14. Bogenrieder T, Herlyn M. Axis of evil: Molecular mechanisms of cancer metastasis. Oncogene. 2003;22:65246536.

15. Madkour BS; Bekheet IW, El Baz AG, Ghobashy S, El-Ganzory H, Essawy FM. Prognostic and Predictive Significance of Haemostatic and Angiogenic Parameters in Cancer Bladder Patients. J Am Sci. 2010;6(11):1091-1097.

16. Angenete E, Langenskiold M, Palmgren I, Falk P, Oresland $\mathrm{T}$, Ivarsson ML. uPA and PAI-1 in rectal cancer-relationship to radiotherapy and clinical outcome. J Surg Res. 2009;153(1):46-53.

17. Langenskiold M, Holmdahl L, Angenete E, Falk P, Nordgren S, Ivarsson ML. Differential prognostic impact of UPA and PAI-1 in colon and rectal cancer. Tumour Biol. 2009;30(4):210-220.

18. Suega K, Bakta IM. Correlation Between Clinical Stage of Solid Tumor and D Dimer as a Marker of Coagulation Activation. Acta Med Indones. 2011;43(3):162-167.

19. Benoy IH, Salgado R, Elst H, Van Dam P, Weyler J, Van Marck E et al. Relative microvessel area of the primary tumour, and not lymph node status, predicts the presence of bone marrow micrometastases detected by reverse transcriptase polymerase chain reaction in patients with clinically non-metastatic breast cancer. Breast Cancer Res. 2005;7:210-221.

20. Xu G, Zhang YL, Huang W. Relationship between plasma D-dimer levels and clinicopathologic parameters in resectable colorectal cancer patients. World J Gastroenterol. 2004;10(6):922-923.

21. Kwon HC, Oh SY, Lee S. Plasma Levels of Prothrombin Fragment F1+2, D-dimer and Prothrombin Time Correlate with Clinical Stage and Lymph Node Metastasis in Operable Gastric Cancer Patients. Jpn J Clin Oncol. 2008;38(1):2-7

*Corresponding author:

Dr Rashmi C, \#1, Tata Sherwood road, Shankarappa Reddy gardens, Basavanagara, Bangalore-560037, India

Phone: +91 9663652447

Email: drrashmireddy@gmail.com

Financial or other Competing Interests: None. 